\title{
TOPOSES ARE SYMMETRIC MONOIDAL CLOSED CATEGORIES
}

\author{
Viliam Slodičák \\ ${ }^{I}$ Department of Computers and Informatics Faculty of Electrical Engineering and Informatics \\ Technical University of Košice, Košice, Slovak Republic \\ viliam.slodicak@tuke.sk
}

\begin{abstract}
The category theory provides possibilities to model many important features of computer science. We used the symmetric monoidal closed category for the construction of a model of linear type theory. Toposes as specific categories make it possible to model theories over types. In this article we prove that topos is symmetric monoidal closed category. This fact will allow us to use topos in the role of symmetric monoidal closed category - for construction of the models of the type theory.
\end{abstract}

\section{Introduction}

The objective of our article is to show that toposes have properties of symmetric monoidal closed categories. Toposes are special categories - cartesian closed categories with some extra structure which produce an object of subobject for each object [1]. This structure makes toposes more like the category of sets than cartesian closed categories generally are. Toposes have proved attractive for the purpose of modeling computations. If we want a topos to be a generalized mathematical theory, we suppose that a set of hypotheses or axioms are formulated in predicate logic. They implicitly define some kind of structure of a general theory defined by axioms formulated possibly in higher-order logic [2]. An elementary topos is one whose axioms are formulated in the first-order logic, i.e. an elementary topos is the generalized axiomatic set theory [3]. On the other hand, toposes have many important properties for expressing and modeling the logic. The category-theoretic notion of a topos is called upon to study the syntax and semantics of higher-order logic, too. Then syntactical systems of logic are replaced by toposes and models by functors on those toposes. We constructed a model of first-order linear logic [4]; we also introduced syntax of basic logical language and showed one of the ways of interpreting the semantics of that language in topos [5].The description of program behavior based on coalgebras in toposes we introduced in [6]. The language of linear logic can be also very suitable for behavior of semantic web [7]. 
In the literature there are many approaches to the semantics of linear logic. The first and the simplest is the phase semantics by Girard [8]; further there are consequence algebras, quantales [9], coherence spaces, resource semantics [10] and many others. We constructed the model also in symmetric monoidal closed category as semantics for introduced linear logic because for any symmetric monoidal closed category there is a linear type theory whose model is this category $[11,12]$.

In [4] we constructed the model of first-order linear logic and model of the higher-order linear logic without exponentials in toposes. We showed a simpler way to construct those models. Because toposes are very useful for expressing the linear logic, we would like to extend our model for the exponential operators of linear logic, as well. For this purpose we want to show that topos is also a symmetric monoidal closed category, so in our approach we would be able to use the properties of symmetric monoidal closed categories for expressing the exponential operators.

\section{Basic notions}

In this section we briefly introduce notions of symmetric monoidal closed categories and toposes.

\subsection{Category theory}

A category $\mathrm{C}$ is a mathematical structure consisting of objects, e.g. $A, B, \ldots$ and morphisms of the form $f: A \rightarrow B$ between them. Every object has the identity morphism $i d_{A}: A \rightarrow A$ and morphisms are composable. Because the objects of category can be arbitrary structures, categories are useful in computer science, where we often use more complex structures not expressible by sets. Morphisms between categories are called functors, e.g. a functor $F: C \rightarrow D$ from a category $C$ into a category $D$ is a morphism which preserves the structure.

\subsection{Symmetric monoidal closed categories}

Symmetric monoidal closed categories are special categories which have been used for expressing the semantics of linear logic $[2,11]$. There are also other approaches, like phase semantics, consequence algebras, quantales, coherence spaces, resource semantics and many others.

In our approach we prefer symmetric monoidal closed category as semantics for intuitionistic linear logic because symmetric monoidal closed categories are 
a generalization of type representation in cartesian closed categories. It also holds that every consequence algebra and quantale is a symmetric monoidal closed category when they are viewed as categories, and for any symmetric monoidal closed category there is a linear type theory whose model is this category.

A monoidal category $(\mathrm{C}, \otimes I, a, l, r)$ (or $\mathrm{C}$ for simple) consists of:

- a category $\mathrm{C}$;

- a tensor functor $\otimes: \mathrm{C} \otimes \mathrm{C} \rightarrow \mathrm{C}$;

- natural isomorphisms $a, l, r$ of the form

$$
\begin{aligned}
& a_{A, B, C}:(A \otimes B) \otimes C \rightarrow A \otimes(B \otimes C), \\
& l_{A}: I \otimes A \rightarrow A, \\
& r_{A}: A \otimes I \rightarrow A,
\end{aligned}
$$

where $A, B, C, D$ are objects of category $C$. The first isomorphism express the associativity of tensor functor, the two latter the left and right neutral element of it. They also have to satisfy the coherence axioms expressed by the following commutative diagrams at Figure 1 (the triangle) and at Figure 2 (the pentagon).

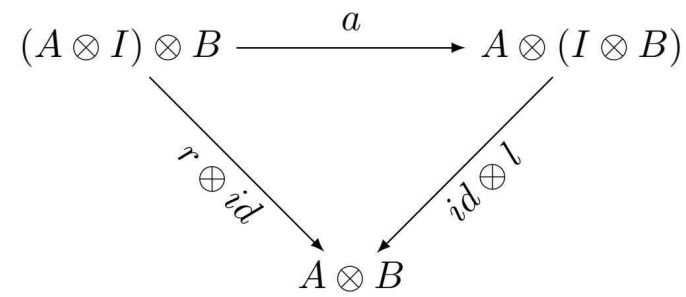

Fig. 1. Triangle - coherence axiom for the isomorphisms $l$ and $r$ in monoidal category

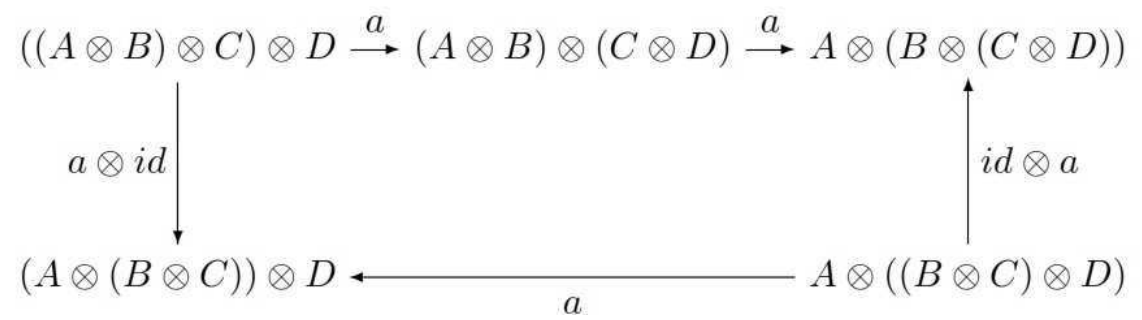

Fig. 2. Pentagon - coherence axiom for the isomorphism $a$

For achieving the commutativity of the tensor product we extend the monoidal category with a natural isomorphism

$$
c_{A, B}: A \otimes B \rightarrow A \otimes B,
$$


that satisfies the coherence axiom at Figures 3 and 4.

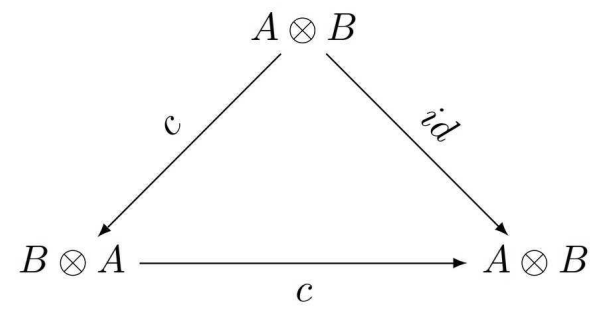

Fig. 3. Coherence axiom for $c$ isomorphism in monoidal category

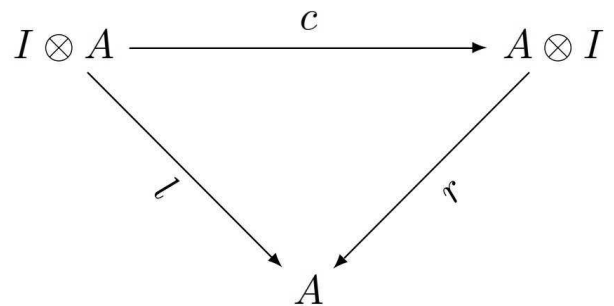

Fig. 4. Coherence axiom for $c, l$ and $r$ isomorphisms in monoidal category

A symmetric monoidal closed category $\mathrm{C}$ is closed, if the functor $-\otimes A$ for every object $A$ in $\mathrm{C}$ has a specified right adjoint - the hom functor $\operatorname{Hom}(A,-)$

$$
-\otimes A-\mid \operatorname{Hom}(A,-),
$$

such that there exist natural transformations

$$
\begin{gathered}
\varepsilon_{A, B}: \operatorname{Hom}(A, B) \otimes A \rightarrow B \\
\delta_{A, B}: A \rightarrow \operatorname{Hom}(B, A \otimes B)
\end{gathered}
$$

which satisfy the triangle identities for an adjunction:

$$
i d=\left(\delta \otimes i d_{B}\right) \circ \varepsilon: A \otimes B \rightarrow \operatorname{Hom}(B, A \otimes B) \otimes B \rightarrow A \otimes B
$$

and

$$
i d=\delta \circ \operatorname{Hom}\left(i d_{A}, \varepsilon\right): \operatorname{Hom}(A, B) \rightarrow \operatorname{Hom}(A, \operatorname{Hom}(A, B) \otimes A) \rightarrow \operatorname{Hom}(A, B) .
$$

In [13] we have defined linear type theory and its interpretation in category. The interpretation of linear type theory we constructed in the symmetric monoidal closed category as a pair of functions $(i, j)$ :

$$
(i, j): \operatorname{LinTT}(B, F, E) \rightarrow(\mathbf{C}, \otimes, I, a, l, c, \operatorname{Hom}(-,-))
$$

where $B$ is the set of basic types, $F$ is the set of function symbols and $E$ is the set of axioms. Then 


$$
i: B \rightarrow O b(\mathrm{C})
$$

is the type interpretation function, and

$$
j(f): i(A) \rightarrow i(B)
$$

is the function interpretation mapping (defined for the function symbol $f \in F$ of the form $f: A \rightarrow B$ ).

For example, if $C$ is a category with finite products then the tensor product ' $\otimes$ ' is given by Cartesian category product, $I$ is a terminal object of category $C$ and natural isomorphisms are expressed by appropriate combinations of projection morphisms and pairing.

\subsection{Toposes}

Toposes are special kind of category defined by axioms saying roughly that certain construction one can make with sets can be done in category theory [12]. A topos is a category $E$ which satisfies the following properties:

1. E has a terminal object $\mathbf{1}$, and for every corner of morphisms $X \rightarrow Z \leftarrow Y$ in E there is a pullback (Figure 5).

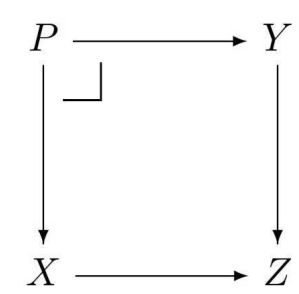

Fig. 5. Pullback in topos

2. E has a subobject classifier: an object (traditionally denoted) $\Omega$ with a monomorphism true $: 1 \rightarrow \Omega$ such that for any morphism $m: M>\rightarrow X$ in $\mathrm{E}$ there is a unique morphism $\chi_{m}: X \rightarrow \Omega$ such that the diagram at Figure 6 is a pullback.

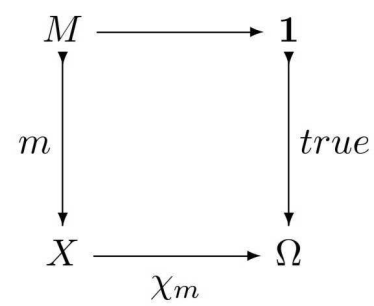

Fig. 6. Subobject classifier in topos 
3. $\mathrm{E}$ has power objects: for each object $X$ in $\mathrm{E}$, an object $\Omega^{X}$ and a morphism eval $_{X}: X \times \Omega^{X} \rightarrow \Omega$ such that for any morphism $f: X \times Y \rightarrow \Omega$ in E there is a unique morphism $\lambda_{X} \cdot f: Y \rightarrow \Omega^{X}$ such that the diagram at Figure 7 commutes.

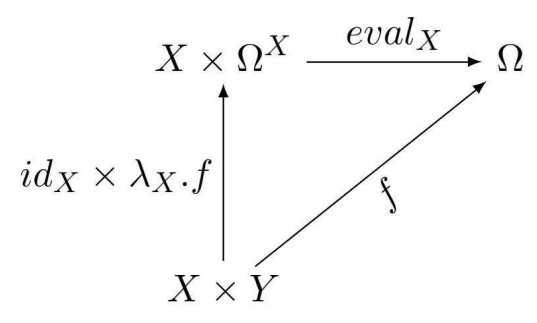

Fig. 7. Evaluation morphism in topos

By 1) a topos has all finite limits. In particular, the product $X \times Y$ of two objects $X$ and $Y$ is the pullback of the corner of morphisms $X \rightarrow \mathbf{1} \leftarrow Y$. For each object $X$, the unique morphism to the terminal object 1 we denote $!_{X}: X \rightarrow \mathbf{1}$. The morphism $\chi_{m}: X \rightarrow \Omega$ of 2 ) is called the evaluation characteristic (or classifying) morphism of the monomorphism $m: M>\rightarrow X$. In 3), the morphism eval $_{X}: X \times \Omega^{X} \rightarrow \Omega$ is called evaluation, and the morphism $\lambda_{X} . f: Y \rightarrow \Omega^{X}$ is called the $X$-transpose of $f: X \times Y \rightarrow \Omega$. The object $\Omega^{X}$ is the exponential of $\Omega$ by $X$. For each object $X$ we have an exponential functor $\operatorname{Hom}(-, X): \mathrm{E} \rightarrow \mathrm{E}$ which is $\mathrm{s}$ right adjoint to the functor $-\times X$, so the topos is a Cartesian closed category [3].

\section{Topos is symmetric monoidal closed category}

We constructed a model of multiplicative fragment $(L, 1, \otimes,-\circ)$ of linear logic in topos. We would like to extend this model by exponential operators of course (!) and why not (?). We need to express the linear exponential operators in linear exponential monad which is constructed over the topos. The linear exponential monad is usually constructed over symmetric monoidal closed category.

Our objective is to sow that topos has properties of symmetric monoidal closed category because we would like to extend our model of linear logic by exponential operators constructed in topos.

Topos has a subobject classifier. It is a special object $\Omega$ of a category together with monomorphism true. A subobject classifier classifies subobjects of a given object according to which elements belong to the subobject. Because of this role, 
the subobject classifier is also referred to as the truth value object [14]. In fact the way in which the subobject classifier classifies subobjects of a given object, is by assigning the values true to elements belonging to the subobject in question, and false to elements not belonging to the subobject. This is the way the subobject classifier is widely used in the categorical description of logic.

Now we formulate the proof, that topos is also a symmetric monoidal closed category. This property is important when we want to construct a model of linear logic as topos. So we formulate the proposition: A topos is symmetric monoidal closed category.

Proof. Let $\mathrm{E}$ be the topos. We have to prove that $\mathrm{E}$ is the symmetric monoidal closed category. So we have to prove that $\mathrm{E}$ satisfies the definition of symmetric monoidal closed category.

Category $\mathrm{C}$ in definition of symmetric monoidal closed category is a Cartesian closed category [11]. According to the definition of topos is E also Cartesian closed category.

Tensor product ' $\otimes$ ' has a form $\otimes: C \otimes C \rightarrow C$. Terminal object $I$ of category $C$ is neutral element of the tensor product.

A product ' $x$ ' in topos corresponds to the tensor functor:

$$
x: E \times E \rightarrow E .
$$

The product has neutral element 1 which is the terminal object of topos $E$ [3]. Tensor product is associative [11]. The category $\mathrm{C}$ has the isomorphism expressing the associativity of tensor product $a_{A, B, C}$. As the topos is cartesian closed category, from the properties of product and from the definition of category, we formulate the associativity in topos as an isomorphism $\operatorname{assoc}_{X, Y, Z}$ by the following way:

$$
\operatorname{assoc}_{X, Y, Z}:(X \times Y) \times Z \rightarrow X \times(Y \times Z) .
$$

The left and right neutral element of the tensor product is given by isomorphisms $l_{A}$ and $r_{A}$. In topos $\mathrm{E}$ from the properties of product we define isomorphisms

$$
\begin{aligned}
& \text { left }_{X}: \mathbf{1} \times X \rightarrow X, \\
& \text { right }_{X}: X \times \mathbf{1} \rightarrow X .
\end{aligned}
$$

The following equations also hold:

$$
\begin{aligned}
& \text { left }_{X}=\pi_{2}(\mathbf{1} \times X), \\
& \text { right }_{X}=\pi_{1}(X \times \mathbf{1}),
\end{aligned}
$$


where $\pi_{1}(-)$ and $\pi_{2}(-)$ are the first and second projection morphisms:

$$
\begin{aligned}
& \pi_{1}: A \times B \rightarrow A, \\
& \pi_{2}: A \times B \rightarrow B .
\end{aligned}
$$

The commutativity in the category $\mathrm{C}$ is expressed by the isomorphism $c_{X, Y}$. For any pair of objects $X, Y \in O b(\mathrm{C})$ we define an isomorphism change $_{X, Y}$ as follows: while topos is cartesian closed category, we define an object $X \times Y \in O b$ (C) together with projections $\pi_{1}(X \times Y)=X$ and $\pi_{2}(X \times Y)=Y$. While it holds that

$$
\operatorname{dom}\left(\pi_{1}\right)=\operatorname{dom}\left(\pi_{2}\right)=X \times Y,
$$

we formulate change $e_{X, Y}$ isomorphism as a product function

$$
\text { change }_{X, Y}=\left\langle\pi_{2}, \pi_{1}\right\rangle: X \times Y \rightarrow X \times Y
$$

that expresses the commutativity in topos.

The category $\mathrm{C}$ is closed if for every object $A \in O b(\mathrm{C})$ the functor $-\otimes A$ has a specified right adjoint, the hom functor $\operatorname{Hom}(A,-)$,

$$
-\otimes A-\mid \operatorname{Hom}(A,-)
$$

with the natural transformations $\varepsilon_{A, B}$ and $\delta_{A, B}$.

Similarly we define closeness for topos: for every object $X \in O b(\mathrm{E})$ the functor $-\times X$ has the right adjoint, the hom-functor $\operatorname{Hom}(X,-)$ :

$$
-\times X-\mid \operatorname{Hom}(X,-),
$$

with the natural transformations

$$
\begin{aligned}
& \operatorname{eval}_{X}: \Omega^{X} \times X \rightarrow \Omega, \\
& D_{X}: X \rightarrow \operatorname{Hom}(\Omega, X \times \Omega),
\end{aligned}
$$

which also satisfy the triangle identities for an adjunction at Figures 8 and 9:

$$
i d_{X \times \Omega}=\left(D_{X} \times i d_{\Omega}\right) \operatorname{eval}_{X \times \Omega}: X \times \Omega \rightarrow \operatorname{Hom}(\Omega, X \times \Omega) \times \Omega \rightarrow X \times \Omega
$$

and

$i d=D_{\operatorname{Hom}(X, \Omega)} \operatorname{Hom}\left(i d_{X}, \operatorname{eval}_{X}\right): \operatorname{Hom}(X, \Omega) \rightarrow \operatorname{Hom}(X, \operatorname{Hom}(X, \Omega) \times X) \rightarrow \operatorname{Hom}(X, \Omega)$. 


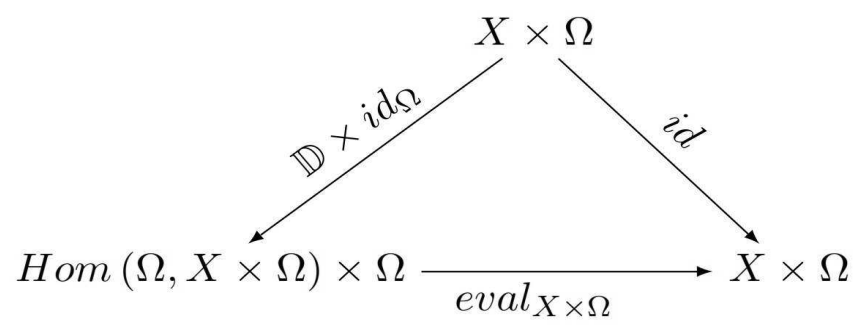

Fig. 8. Triangle identity for adjunction

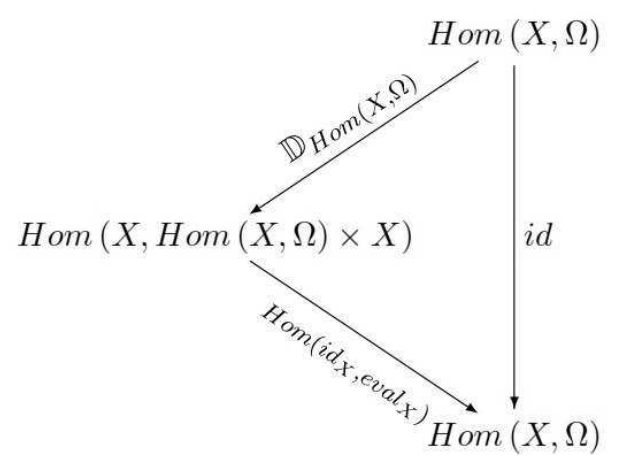

Fig. 9. Triangle identity for adjunction

We have shown how the topos satisfies the definition of symmetric monoidal closed category. So we can conclude, that any topos E is symmetric monoidal closed category given as a structure

$$
(\mathrm{E}, \times, \mathbf{1}, \text { assoc, left, right }, \operatorname{Hom}(-,-)) \text {. }
$$

\section{Conclusions}

In our paper we formulated basic aspects about the topos theory needed for our approach of scientific problem solving. We presented a proof that topos has properties of symmetric monoidal closed categories. Our next goal will be the construction of model of linear logic with its exponential operators of course and why not by using that property of topos.

\section{Acknowledgement}

This work has been supported by KEGA Grant No. 050TUKE-4/2012: Application of Virtual Reality Technologies in Teaching Formal Methods. 


\section{Zusammenfassung}

Die Theorie von Kategorien bietet viele wichtige Möglichkeiten für die theoretische Informatik. Die Topoi sind spezielle Kategorien, die ermöglichen, die Theorien über den Typen zu modellieren. In unserem Artikel arbeiten wir mit der symmetrischen monoidalen verschlossenen Kategorie für das Konstruieren vom Modell der linearen Kategorie von Typen. Wir beweisen, dass der Topos eine symmetrische monoidale verschlosene Kategorie ist, also es ist möglich, ihn für das Konstruieren von Modell der linearen Theorie von Typen zu verwenden.

\section{References}

[1] Barr M., Wells C., Category Theory for Computing Science, Prentice Hall International, 1990.

[2] Novitzká V., Slodičák V., Categorical Structures and their Application in Informatics, Equilibria, Košice 2010.

[3] Barr M., Wells C., Toposes, Triples and Theories, Springer-Verlag, 2002.

[4] Slodičák V., The Rôle of Toposes in the Informatics, PhD. Thesis, Technical University of Košice, Slovakia, 2008 (in Slovak).

[5] Novitzká V., Slodičák V., Verbová A., On modelling higher-order logic, Informatics 2007, Proceedings of the Ninth International Conference on Informatics, Slovak Society for Applied Cybernetics and Informatics, Bratislava 2007, 156-162.

[6] Slodičák V., Szabó Cs., Recursive coalgebras in mathematical theory of programming, The $8^{\text {th }}$ Joint Conference of Mathematics and Computer Science, Győr, Hungary, Novadat Bt. 2010, 385-394.

[7] Šaloun P., Velart Z., Nekula J., Towards automated navigation over multilingual content. Semantic Hyper/Multi-media Adaptation: Schemes and Applications. Studies in Computational Intelligence, Springer 2012.

[8] Girard J., Linear Logic: Its syntax and semantics, Cambridge University Press, 2003.

[9] Mulvey C. J., "\&”, Suppl. Ai Rend. Del Circ. Mat. Di Palermo, Serie II, 1986.

[10] Dam M., Relevance logic and concurrent composition, $\mathrm{PhD}$. thesis, University of Edinburgh, 1990.

[11] Ambler S.-J., First order linear logic in symmetric monoidal closed categories, PhD. thesis, University of Edinburg, 1991.

[12] Novitzká V., Logical reasoning about programming of mathematical machines, Acta Electrotechnica et Informatica, Košice 2005, 50-55.

[13] Novitzká V., Mihályi D., Slodičák V., Linear logical reasoning on programming, Acta Electrotechnica et Informatica, Košice 2006, 34-39.

[14] Taylor P., Practical Foundations of Mathematics, Cambridge University Press, 1999. 